\title{
Acceleration for a High Energy Mon Collider
}

\author{
J. Scott Berg \\ Brookhaven National Laboratory
}

January 2000 


\section{Acceleration for a High Energy Muon Collider}

J. Scott Berg

Broothaven National Laboratory; Building 901A; PO Box 5000; Upton, NY 11973-5000

Abstract. We describe a method for designing the acceleration systems for a muon collider with particular application and examples for a high energy muon collider. This paper will transverse issues will be brieffy discussed.

\section{INTRODUCTION}

The cost of a high energy muon collider will be clearly be dominated by the cost of accelerating the muons to their maximum energy. It is thus important to study possible texhiniques for acceleration, the ardvantages and disadvantages of these techniques, and how to perform a cost optimization of a final design.

The acceleration of muons poses various challenges that that are not present in the acceleration of other types of charged particles. Since muons have a finite lifetime (approximately 1000 turns), one cannot take a long time to accelerate them, so traditional accelerating synchrotrons such as are used for protons cannot be used. Due to the difficulty of cooling the muon beam, the longitudinal emittances tend to be large (as high as $0.047 \mathrm{eV}$-s), and this ends up making the acceleration significantly more challenging, especially at lower energies.

The larger mass of the muons (as compared to electrons) prevents them from emitting substantial amounts of synchrotron radiation, and thus there is nothing preventing them from being bent in an arc. While a conventional synchrotron will not work, a recirculating accelerator is certainly possible. An additional advantage of a recirculating accelerator is that it potentially gives high RF-to-beam power efficiencies, since the same linac can be used for multiple passes through the system.

\section{Design Parameters}

Table 1 gives parameters for high energy inuon colliders that are relevant to the design of the acceleration systems $[1,2]$. In that table, $p_{\text {min }}$ is the momentum at which acceleration begins, $p_{\max }$ is the momentun in the collider, $\epsilon_{L}$ is the longitu-
TABLE 1. Parameters for various high energy muon colliders.

\begin{tabular}{|c|c|c|c|c|}
\hline $\begin{array}{c}P_{\text {minum }} \\
\mathrm{MoV} / \mathrm{c}\end{array}$ & $\mathrm{TeV} / \mathrm{c}_{\mathrm{Pmax}}$ & $\begin{aligned} \ell L \\
m e V-a\end{aligned}$ & $\underset{\mu \mathrm{m}}{\boldsymbol{c}_{\mathrm{m}}}$ & $\begin{array}{r}N \\
10^{12} \\
\end{array}$ \\
\hline 186 & 0.5 & 24 & 50 & 4 \\
\hline 186 & 10 & 21 & 38 & 3 \\
\hline 186 & 100 & 47 & 8.7 & 0.8 \\
\hline
\end{tabular}

dinal emittance (normalized), $\epsilon_{n}$ is the normalized transverse emittance, and $N$ is the number of particles per bunch.

We will assume that maximum accelerating gradients and the $r_{0} / Q$ of the cavities scale with frequency according to

$$
v=30 \mathrm{MV} / \mathrm{m} \sqrt{\frac{f}{800 \mathrm{MHz}}} \quad \frac{r_{s}}{Q}=1000 \Omega / \mathrm{m} \frac{f}{800 \mathrm{MHz}},
$$

where $f$ is the RF frequency.

\section{METHODS FOR COMPUTING PARAMETERS}

This paper will primarily addrees how to compute longitudinal parameters, but will include very rough estimates of transverse parameters for the design. There are really two types of aystem that will be considered: straight linacs and recirculuting accelerators. Straight linacs are used for acceleration from the lowest energies, since the relative energy spreads at lower energies will be impossible to get through a conventional arc. Once the beam reaches a sufficient energy, however, recirculating accelerators will be used.

\section{Straight Linacs}

At the lowest energies, the velocity of the particles cannot be considered to be constant, and thus it is probably best not to accelerate on-crest; instead, one should allow particles to undergo synchrotron oscillations, with the time-of-flight variation coming purely from the velocity variation with energy.

As a first approximation to the behavior of synchrotron motion in the linacs, we can take an adiabatic approximation wherein the RF bucket is determined by the sinusoidal RF fields and the velocity variation of the muons with energy. The smallness parameter in the adiabatic approximation is the quantity

$$
\frac{1}{k_{\mathrm{a}} p} \frac{d p}{d s}
$$

where $k_{\text {a }}$ is the synchrotron wave number (computed later), $p$ is the muon momentum for the reference particle, and $s$ is the distance along the reference orbit. It 
will turn out that this quantity is not in fact very small; however, lacking a better analytic description, one can nonetheless use the results under the adiabatic approximation to give a first (probably optimistic) guess as to what the parameters may be.

One could consider running on-crest at some point during the acceleration process, particularly once the adiabatic approximation becomes particularly bad. This will first of all necessarily lead to some longitudinal emittance growth. Furthermore, it will be necessary to introduce some momentum compaction to counteract the effect of the velocity variation with energy, but doing that for a beam with the relative energy spreads that this beam will have would be highly nontrivial.

\section{Hamiltonian Description}

The Hamiltonian describing longitudinal particle motion in the linac is

$$
\begin{aligned}
& -\frac{1}{c} \sqrt{\left[E_{0}(s)+\Delta\right]^{2}-\left(m c^{2}\right)^{2}}+\frac{E_{0}(s) \Delta}{p_{0}(s) c^{2}}+p_{0}(s) \\
& \quad+\frac{q v\left(s, t_{0}(s)+\tau\right)}{\omega}\left[\sin \left(\omega \mid t_{0}(s)+\tau\right]+\phi\left(s, t_{0}(s)+\tau\right)\right) \\
& \left.-\omega \tau \cos \left(\omega t_{0}(s)+\phi\left(s, t_{0}(s)+\tau\right)\right)-\sin \left(\omega t_{0}(s)+\phi\left(s, t_{0}(s)+\tau\right)\right)\right]
\end{aligned}
$$

where $E_{0}(s)$ is the energy of the reference particle, $p_{0}(s)=\sqrt{E_{0}^{2}(s)-\left(m c^{2}\right)^{2}}$ is its momentum, and $t_{0}$ is its arrival time at longitudinal position $s$ in the linac. The particles have mass in and charge $q$. The gradient and phase of the fundamental mode with frequency $\omega$ at longitudinal position $s$ and time $t$ are $v(s, t)$ and $\phi(s, t)$ respertively. These quantities are related through

$$
\frac{d t_{0}}{d s}=\frac{E_{0}(s)}{p_{0}(s) c} \quad \frac{d E_{0}}{d s}=q v\left(s, t_{0}(s)\right) \cos \left(\omega t_{0}(s)+\phi\left(s, t_{0}(s)\right)\right)
$$

The canonical coordinate is $\tau$ for the deviation of the arrival time at a given longitudinal position $s$ of a particle from that of a reference particle, and the canonical unomentum is $-\Delta$, where $\Delta$ is the deviation of the energy of a particle from that of the reference particle. The Hamiltonian ignores effects of beam loading, and only considers the effect of the fundamental mode in the linac.

The reason for putting time dependence in $v$ and $\phi$ is to take into account power input, wall losses, and in principle beam loading (although the latter really require a self-consistent solution, but the average effect could be put in). Except for beam loading, these time variations occur over time scales which are long compared to the time that it takes the bunch to pass by a given point in the linac. Thus, for the purposes of this discussion, the time dependence will be ignored.
It will be convenient to define an effective phase of $\psi(s) \equiv \omega t_{0}(s)+\phi(s)$. The adiabatic approximation is really an assumption that $v(s)$ and $\psi(s)$ change very slowly. "Very slowly" must be in relation to some other time scale, and it turns out that this time scale is really defined by the quantity (2) being small (this will not be demonstrated here). The adiabatic approximation is computed by performing calculations as if $v(s)$ and $\psi(s)$ were in fact constant. The idea is that synchrotron oecillations are occurring so rapidly the $v(s)$ and $\psi(s)$ really are nearly constant over the period of a synchrotron oscillation.

There are of course variations in $v(s)$ due to the fact that the fields amplitudes not constant over the length of a cavity cell. For these variations, one can make another adiabaticity argument: as long as the variation in particle energy and arrival time offet is small over the length of a cavity cell, the average value of $v(s)$ can be used.

Thus, these two adiabaticity arguments allow us to remove the $s$ dependence from $v$ and $\psi$ in subsequent discussions, and the results will be valid to the extent that the adiabaticity arguments are valid.

\section{Linearization}

There are two periodic sets of fixed points of the above Hamiltonian: one set with $\Delta=0$ and $\omega \tau=2 \pi m$, the other set at $\Delta=0$ and $\omega \tau=2 \pi m-2 \psi$. As long as $\psi<0$, the fixed point at $\tau=0$ is stable. Linearizing the Hamiltonian about that fixed point, we get

$$
\frac{1}{2} \frac{\left(m c^{2}\right)^{2}}{c\left(p_{0} c\right)^{3}} \Delta^{2}-\frac{1}{2} q v \omega \sin \psi \tau^{2}
$$

From this, we can compute the square of the aspect ratio of a matched beam to be

$$
\frac{\sigma_{\Delta}^{2}}{\sigma_{\tau}^{2}}=-\frac{q c v \omega\left(p_{0} c\right)^{3} \sin \psi}{\left(m c^{2}\right)^{2}}
$$

and the square of the synchrotron wave number $k_{s}(2 \pi$ divided by the synchrotron wavelength) to be

$$
k_{*}^{2}=-\frac{q v \omega\left(m c^{2}\right)^{2} \sin \psi}{c\left(p_{0} c\right)^{3}} .
$$

Here $\sigma_{\Delta}$ is the RMS energy spread, and $\sigma_{r}$ is the RMS bunch length in arrival time units.

\section{Bucket Area}

The Hamiltonian (3) can be used to find an equation describing the separatrix of the RF bucket. The separatrix contains the unstable fixed point at $\omega \tau=-2 \psi$, 
and the Hamiltonian has a value of

$$
\frac{2 q v}{\omega}(\psi \cos \psi-\sin \psi)
$$

at that point and therefore along the separatrix. The separatrix is at $\tau$ has the energy deviation values

$$
\begin{gathered}
\Delta=E_{0} w \pm p_{0} c \sqrt{2 w+w^{2}} \\
w=\frac{q v p_{0} c^{2}}{\left(m c^{2}\right)^{2} \omega}[(2 \psi+\omega \tau) \cos \psi-\sin (\psi+\omega \tau)-\sin \psi]
\end{gathered}
$$

From this, the half-width of the bucket can be computed to be

$$
2 p_{v} c \sqrt{\frac{q v p_{0} c^{2}}{\left(m c^{2}\right)^{2} \omega}} \sqrt{\psi \cos \psi-\sin \psi} \sqrt{1+\frac{q v p_{0} c^{2}}{\left(m c^{2}\right)^{2} \omega}(\psi \cos \psi-\sin \psi)} .
$$

Now one might want to ask how large a bunch the bucket can hold. This should really be computed by computing the area of the RF bucket directly. However, a simpler method can be used which is approximately correct. Take the bucket a simpler method can be used which is approximately correct. Take the bucket
half-width to be $k \sigma_{\Delta}$, where $k$ is an arbitrary factor indicating how full you would like the bucket to be. This gives an expression for $\sigma_{\Delta}$. Next, use the aspect ratio (6) to compute $\sigma_{\tau}$ in terms of $\sigma_{\Delta}$. The product of $\sigma_{\tau}$ and $\sigma_{\Delta}$ is the longitudinal emittance $\epsilon_{L}$, and thus we have another expression for $\sigma_{\Delta}$. Equating these two expressions for $\sigma_{\Delta}$, we get a relationship between the longitudinal emittance that the bucket will hold and the bucket and beam parameters. The result is

$$
\frac{4}{k^{2}} \frac{p_{0} c}{\omega} \sqrt{\frac{q v p_{0} c^{2}}{\left(m c^{2}\right)^{2} \omega}} \frac{\psi \cos \psi-\sin \psi}{\sqrt{-\sin \psi}}\left[1+\frac{q v p_{0} c^{2}}{\left(m c^{2}\right)^{2} \omega}(\psi \cos \psi-\sin \psi)\right]=\epsilon_{L}
$$

\section{Decay Losses}

It is well known that for decaying particles at constant velocity, if they travel a distance $s$, the number of particles $N$ at the end of that distance is related to the number $N_{0}$ at the beginning of that distance by

$$
N=N_{0} e^{-o m / p r}
$$

where $\tau$ is the lifetime if the particles in their rest frame and $p$ is the particles momentum.

When the particles are undergoing constant acceleration parallel to their momentun, a calculation is necessary. Integrating

$$
\frac{d N}{d s}=-\frac{m}{p \tau}
$$

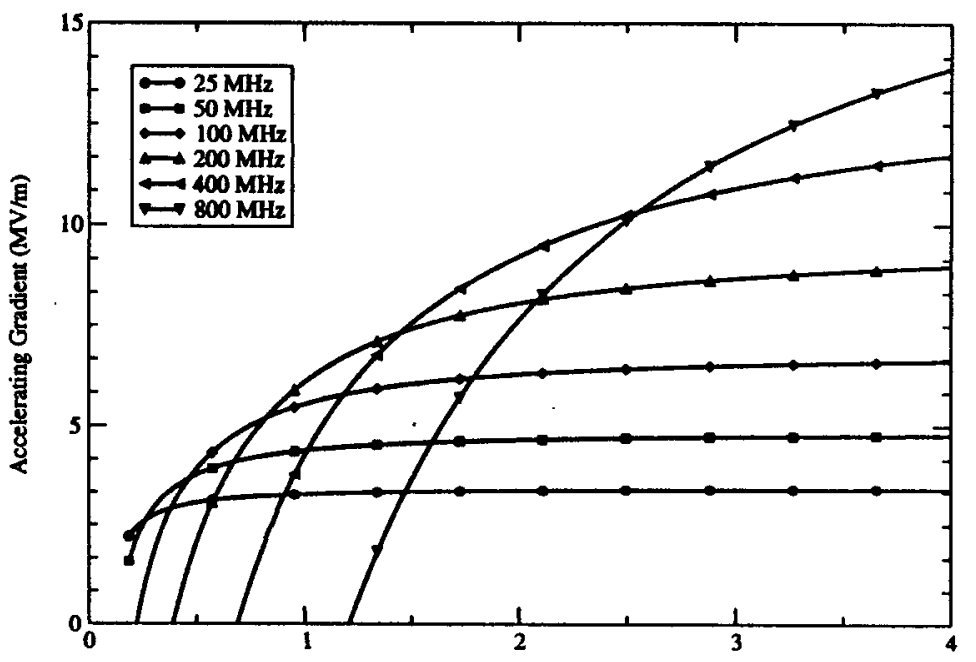

Momentum (GeV/c)

FIGURE 1. Accelerating gradiont as a function of momenta for various RF frequencies, using $0.5 \mathrm{TeV} / \mathrm{c}$ parameters.

when the energy varies according to

$$
\frac{d E}{d s}=q v \cos \psi,
$$

the result is

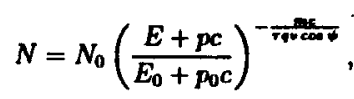

where $E$ is the final energy of the particles, $p$ is their final momentum, and the 0 subscripted numbers are the initial quantities.

In principle there may be corrections that come about from the finite energy spread in the distribution, but these will not be treated here.

\section{Linac Designs}

To apply this to the design of the initial linac for a muon collider, one can take the longitudinal emittance of the beam, choose a value for $k$, choose a frequency 


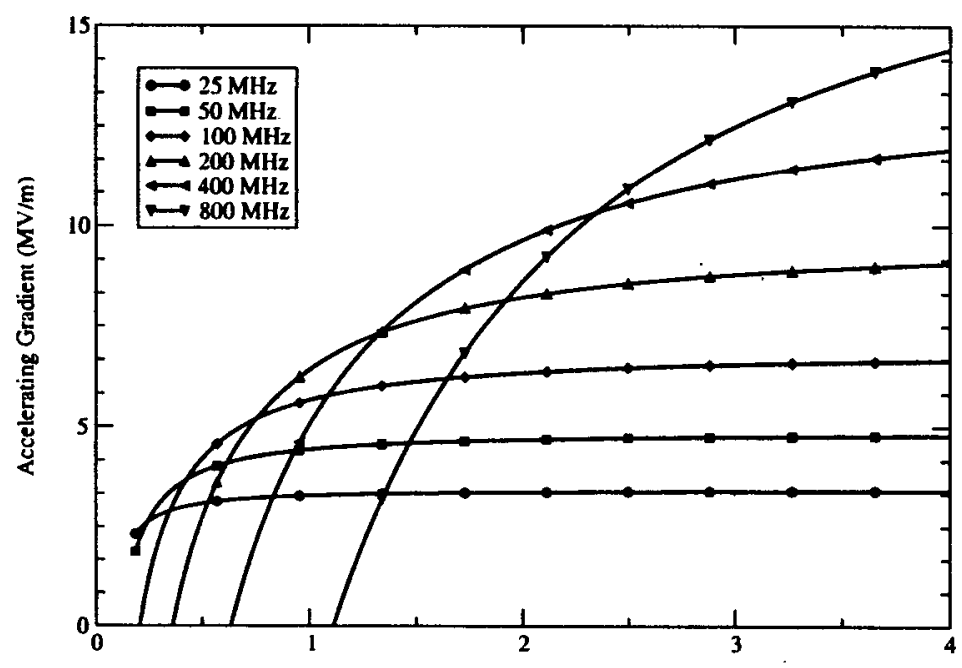

Momentum (GeV/c)

FIGURE 2. Accelerating gradient as a function of momenta for various RF frequencies, using $10 \mathrm{TeV} / \mathrm{c}$ parameters.

$\omega$ and its corresponding gradient $v$, and use (12) to solve for $\psi$ as a function of $p_{0}$. Since the synchronous phase gives the effective accelerating gradient, one thus has the maximum accelerating gradient one can achieve for a given beam emittance and linac parameters as a function of beam momentum. One can plot this for various RF frequencies, and the results are shown in Figs. 1-3. For those figures, a value of 4 was chosen for $k$, and a linac filling factor was assumed to be 0.65 (thus, the maximum average accelerating gradient is really 0.65 times the value from (1)).

Using these plots, one can come up with a scheme for accelerating the bunch in a linac. Assuming that the bunch sliape adiabatically follows the bucket, and that one varies the phase of the RF along the linac to keep the bucket area constant, then Figs. 1-3 really do show the gradient in the linac as a function of reference momentuin. To minimize decay losses, one wants to have the highest gradient possible for a given momentum. Thus, one should switch from one frequency linac to the next when the reference momentum reaches the value where lines for arljacent frequencies cross: One would like to minimize the number of different frequency RF systems used; examining the graph, this suggests that maybe one should choose to jump in frequency by a factor of 4 from one linac to the next.

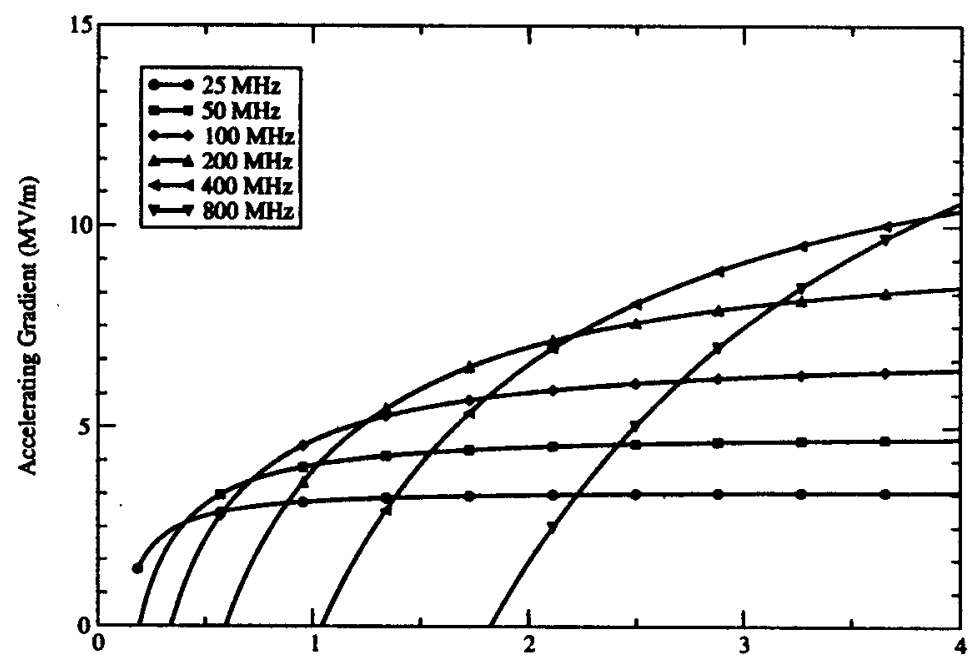

Momentum (GeV/c)

FIGURE 3. Accelerating gradient as a function of moments for various RF frequencies, using $100 \mathrm{TeV} / \mathrm{c}$ parameters.

TABLE 2. Parameters for linacs accelerating to $4 \mathrm{GeV}$.

\begin{tabular}{|c|c|c|c|c|c|c|c|c|}
\hline $\begin{array}{c}P_{\text {mila }} \\
\mathrm{GoV} / \mathrm{c}\end{array}$ & $\begin{array}{c}P_{\text {max }} \\
\mathrm{GoV} / \mathrm{c}\end{array}$ & $\mathrm{Hz}$ & L & pe & $\sigma_{r, \text { out }}$ & $\begin{array}{l}\sigma_{\Delta, \mathrm{in}} \\
\mathrm{MeV}\end{array}$ & $\begin{array}{c}\sigma_{\Delta, \text { out }} \\
{ }_{\mathrm{MeV}}\end{array}$ & $\begin{array}{r}\text { Docay } \\
\%\end{array}$ \\
\hline \multicolumn{9}{|c|}{$0.5 \mathrm{LeV} / \mathrm{c}$ Parametera } \\
\hline 0.186 & 0.68 & 50 & 147 & 1232 & 536 & 19 & 45 & 6.5 \\
\hline 0.68 & 2.10 & 200 & 211 & 280 & 136 & 86 & 176 & 2.8 \\
\hline 2.10 & 4.0 & 800 & 166 & 71 & 47 & 336 & 511 & 0.9 \\
\hline \multicolumn{9}{|c|}{$10 \mathrm{TeV} / \mathrm{c}$ Parameters } \\
\hline 0.186 & $\overline{0.62}$ & 50 & 125 & 1159 & 537 & 18 & 39 & $\overline{5.7}$ \\
\hline 0.62 & 1.92 & 200 & 194 & 281 & 136 & 75 & 154 & 2.8 \\
\hline 1.92 & 4.0 & 800 & 177 & 71 & 44 & 294 & 473 & $\begin{array}{l}2.0 \\
1.0\end{array}$ \\
\hline \multicolumn{9}{|c|}{$100 \mathrm{feV} / c$ Parameters } \\
\hline 0.186 & 0.59 & $\overline{25}$ & 162 & 2257 & 1080 & 21 & $\overline{44}$ & $\overline{7.5}$ \\
\hline 0.59 & 1.82 & 100 & 259 & 565 & 275 & 83 & 171 & 4.0 \\
\hline 1.82 & 4.0 & 400 & 257 & 144 & 87 & 326 & 542 & 1.5 \\
\hline
\end{tabular}

Using the graphs, the schemes suggested in Tab. 2 seem optimal.

The choice of a maximum energy for the linacs of $4 \mathrm{GeV}$ is based on the fact that the arcs for a recirculating accelerator are particularly difficult to construct for energies below this, primarily because of the large energy spread and the requirements on the momentum compaction over the energy range that the arc accepts 
[3].

All analysis of the results from Tab. 2 suggests the following:

- The larger longitudinal emittances gives require substantially longer linacs, and require those linacs to be at lower frequencies. This will substantially effect the cost of such systems. The systems become more efficient and less costly if the longitudinal emittance is reduced.

- There must be a longitudinal matching section from one linac to the next. This matching section can in principle use the lower frequency linac itself. However, these matching sections are potentially very long, and may require momentum compaction to be generated using some sort of arc, which would be difficult with these energy spreads.

Furthermore, it turns out that due to the fact that the adiabatic approximation is not very good in this case, the bunch does not in fact re-orient itself in phase space according to what was given in Tab. 2. The linear matching issues can be corrected for, but it is important to study the phase space dynamics to determine what the effective "bucket" is in this case. In addition, the asymmetric shape of the bucket causes problems with matching in the tails of the distribution.

\section{Recirculating Accelerators}

Once the beam can be reasonably expected to pass through an arc, it becomes more efficient to use a recirculating accelerator to accelerate the beam. A recirculating accelerator consists of two (or more) linacs, connected by one or severa arcs. The beam makes several passes through the linacs. This makes more effcient use of the linacs and the RF power, at the cost of more decays and potential coinplexity in the arc design.

\section{Drift-Kick Map for Linac}

In our coordinate system $(\tau,-\Delta)$, we represent the linear map for the arcs (plus the drift behavior in the linacs) as

$$
\left[\begin{array}{cc}
1 & -D \\
0 & 1
\end{array}\right]
$$

and the linear map for the energy kick from the linac as

$$
\left[\begin{array}{cc}
1 & 0 \\
q v L_{\operatorname{lin}} \omega \sin \phi
\end{array}\right]
$$

where $L_{\text {lin }}$ is the length of the linac, and $\phi$ is the RF phase of the reference particle (same convention as previously). $D$ is a parameter which will be discussed and computed later. Thus, the synchrotron tune for the drift-kick pair will be given implicitly by

$$
\sin \pi \nu_{c}=\frac{1}{2} \sqrt{q v L_{110} \omega D \sin \phi}
$$

The minimum (at the center of the arc) RMS bunch length (in arrival time units) will be

$$
\sqrt{\frac{2 \sin \pi \nu_{0}}{q v \bar{L}_{\mathrm{ln}} \omega \sin \phi}} \sqrt{\epsilon_{t} \cos \pi \nu_{s}}
$$

and the maximum length (center of linac) will be

$$
\sqrt{\frac{2 \sin \pi \nu_{t}}{q v L_{\mathrm{lin}} \omega \sin \phi}} \sqrt{\epsilon_{L} \sec \pi \nu_{0}} .
$$

Similarly, the minimum RMS energy spread (center of linac) will be

$$
\sqrt{\frac{q \nu L_{\operatorname{lin}} \omega \sin \phi}{2 \sin \pi \nu_{0}}} \sqrt{\epsilon_{L} \cos \pi \nu_{0}}
$$

and the maximum energy spread (canter of arc) will be

$$
\sqrt{\frac{q v L_{\ln } \omega \sin \phi}{2 \sin \pi \nu_{0}}} \sqrt{\epsilon_{L} \sec \pi \nu_{s}}
$$

\section{Hamiltonian Description}

To obtain an RF bucket, one must construct an averaged s-independent Hamiltonian which behaves as if the accelerating gradient and the time-of-flight variation are occurring simultaneously instead of sequentially. This Hamiltonian should have the correct linear tune (as computed for the linear transfer map described aluve), and correctly represent the nonlinearity in the RF. It can only correctly give the matched beam eilipee at one point, since the matched beam ellipse varies with position in the ring, while a time-independent Hamiltonian has the same matched eilipse everywhere. Thus we choose an "averaged" matched eilipse for the Hamiitonian to represent: this is most easily chosen by replacing the $\cos \pi \nu$, and $\sec \pi \nu$. in $(20)-(23)$ with 1 . The resuiting Hamiitonian is

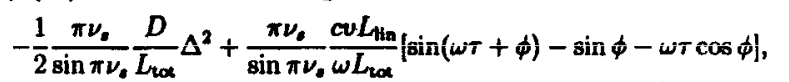

where $L_{\text {int }}$ is $L_{\text {tim }}$ plus the length of the arc (which we will call $L_{\text {arr }}$ ).

Following the same sort of procedure as we did for the linac, we find that the longitudinal emittance accepted by the bucket is

$$
\epsilon_{L}=\frac{2}{k^{2}} \frac{e v L_{\mathrm{lin}}|\sin \phi-\phi \cos \phi|}{\omega \sin \pi \nu_{*}} .
$$




\section{Linac Contribution to D}

There are two contributions to $D$ : one from the arcs, and another from the linacs. The contribution from the arcs is well known and is described by the momentum compaction $\alpha_{C}$. The trick is to characterize the contribution from the linacs. It would be convenient if one could lump the contribution from the linacs in with the contribution from the arcs. This is certainly feasible: if a Hamiltonian can be written as $H_{\Delta}(\Delta)+H_{\tau}(\tau)$, a well-known technique in symplectic integration to get a second-order accurate map is to integrate $H_{\Delta}$ for half a length step, followed by $H_{\mathrm{r}}$ for a full length step, followed by $H_{\Delta}$ for a half step [4]

Thus, we can use the linac Hamiltonian (3), and integrate only the part depending on $\Delta$. Using (4) and taking $v$ and $\phi$ to be constant, we find that after linearizing in $\Delta$,

$$
\tau_{1}-\tau_{0}=\frac{1}{q u c \cos \phi}\left(\frac{1}{\beta_{1}}-\frac{1}{\beta_{0}}\right) \Delta,
$$

where the subscript 0 refers to the beginning of the integration, and the subscript 1 refers to the end. $\beta$ is the speed of the reference particle divided by $c$. There will thus be two contributions from the linacs to $D$ : one from the linac before the arc, where the initial condition in the above integration will be the center of that linac and the final condition will be the end of the linac. Added to that will be a second contribution from the linac after the arc, where the initial condition is the beginning of the linac, and the final condition is the center of that linac. The net result is that there will be a contribution to $D$ which is

$$
\frac{1}{q v c \cos \phi}\left(\frac{1}{\beta_{2}}-\frac{1}{\beta_{0}}\right)
$$

where the subscript 0 refers to the center of the linac before the arc in question, and the subscript 2 refers to the center of the linac that follows the arc.

\section{Arc Parameters}

Given longitudinal design parameters, we now have what we need to specify some basic are parameters. From the previous discussions, $D$ can be written as

$$
D=\frac{4 \sin ^{2} \pi \nu_{s}}{q v L_{\operatorname{lin}} \omega \sin \phi}
$$

But from its basic definition, the definition of $\alpha_{C}$, and the above discussion, it can ulso be written as

$$
\frac{L_{\text {arc }}}{\beta_{1}^{2} p_{1} c^{2}}\left(\alpha_{C}-\frac{1}{\gamma_{1}^{2}}\right)+\frac{1}{q u c \cos \phi}\left(\frac{1}{\beta_{2}}-\frac{1}{\beta_{0}}\right) .
$$

Here the subscript 1 refers to the value in the arc itself, and $\gamma_{1}=1 / \sqrt{1-\beta_{1}^{2}}$. As a result, we have an expression for $\alpha_{C}$ in terms of longitudinal design parameters:

$$
\alpha_{C}=\frac{1}{\gamma_{1}^{2}}+\frac{\beta_{1}^{2} p_{1} c^{2}}{L_{\text {arc }}}\left[\frac{4 \sin ^{2} \pi \nu_{1}}{q v L_{\mathrm{ita}} \omega \sin \phi}+\frac{1}{q v c \cos \phi}\left(\frac{1}{\beta_{0}}-\frac{1}{\beta_{2}}\right)\right]
$$

The vertical RMS beam size is given by

$$
\sigma_{y}=\sqrt{\frac{\beta_{v} \epsilon_{v}}{\beta \gamma}}
$$

where $\beta_{y}$ is the vertical beta-function and $\epsilon_{y}$ is the normalized vertical emittance. Similarly

$$
\sigma_{x}=\sqrt{\frac{\beta_{x} \epsilon_{x}}{\beta \gamma}+\left(\frac{D_{x} \sigma_{\Delta}}{\beta p c}\right)^{2}}
$$

where $D_{x}$ is the horizontal dispersion function. Generally, to compute these values, lattice needs to be laid out. But one can get lower bounds by assuming a constant focusing and bending channel, which would give

$$
\beta_{x}=\beta_{y}=\rho \sqrt{\alpha_{C}} \quad D_{x}=\rho \alpha_{C},
$$

where $\rho$ is the radius of curvature of the arc.

\section{Supplying RF Power}

In this paper, we will assume that power is supplied to the linacs in the recirculator in such a way as to precisely replace the energy removed from the linac by the beam. Such a scheme has the advantage that the longitudinal phase space for the beam can remain matched irrespective of the beam current, assuming that sufficient power is available to make this scheme work for the highest expected beam current. Other schemes, such as one where the stored energy in the linacs is allowed to droop, potentially require that the arcs have different momentum compactions depending on the current in the beam to achieve longitudinal matching potentially making it difficult to run at a current other than the maximum design current.

This paper will make some simple assumptions about how RF power is supplied: there are assumed to be no losses, either through the walls or into loads which are put in for "matching" purposes. The RF simply stores energy into the linacs, and that energy is extracted by the beam. This will necessarily produce the most optimistic values for peak power requirements and efficiencies. More realistic scenarios should be computed at some point. 
Peak power requirements are simple to compute: the beam extracts a certain annount of energy, and that energy must be resupplied by time the beam come around again. One must take into account the fact that there are actually two beams.

Etticiency is important for high energy machines, and this can be computed as follows: the energy supplied to a bean of $N$ particles over $n$ turns through a linac is $n N q v \cos \phi$ per unit length. The energy stored in the linac initially was $v^{2} / \omega\left(r_{d} / Q\right)$ per unit length, and we supplied $N q v \cos \phi$ per unit length $(n-1)$ more times, so the total energy supplied is $v^{2} / \omega\left(r_{s} / Q\right)+(n-1) N q v \cos \phi$. The maximum possible efficiency is therefore

$$
\varepsilon=\frac{n N q v \cos \phi}{(n-1) N q v \cos \phi+\frac{v^{2}}{\omega\left(r_{\lrcorner} / Q\right)}} .
$$

The real efficiency will of course be less than this, due to wall losses and loads plus efticiencies of the devices supplying the RF power.

\section{Recirculator Designs}

If we specify

- The synchrotron tune

- The total energy gain in the recirculator

- The gradient and frequency of the RF

- The number of turns in the recirculator

- The quantity $k$

- The longitudinal emittance

the alove description tells us how to compute the phase at which we should run the RF. While the synchrotron tune may seem like an odd quantity to specify, it in fact makes sense to do so. A high synchrotron tune is advantageous for several reasons:

- It gives a smaller energy spread in the beam (important for simplifying arc design)

- It can minimize collective instabilities

- It can prevent degradation of polarization [5]

However, there is a maximum value for the synchrotron tune, which is about 0.15 per linac-arc pair. The reason for this is that the motion is in fact described by a $s$ dependent Hamiltonian, and not the $s$-independent Hamiltonian (24). The bucket computed for that Hamiltonian is only correct in the limit of small synchrotron tune for the linac-arc pair. For a larger synchrotron tune, the edges of the bucket will degrade until the bucket completely disappears at a synchrotron tune of 0.5
The source of this degradation is the nonlinearity from the RF. This suggests that we try a synchrotron tune of 0.15 per linac-arc pair. For a racetrack design, this corresponds to a one-turn synchrotron tune of 0.3 . The racetrack design seems most efficient in terms of avoid the overhead necessary at the entrance and exit of each linac, but in principle a design with more sides would allow even larger synchrotron tunes.

Using these constraints, we can come up with designs for the recirculators. One can imagine that a collider will be built up by upgrading the machines over time essentially adding recirculating stages. The various machines might have single beam energies of $70 \mathrm{GeV}, 500 \mathrm{GeV}, 10 \mathrm{TeV}$, and $100 \mathrm{TeV}$. Thus, the recirculator stages will have maximum energies at these points.

Because any time spent in arcs is essentially lost (and gives excess decays), one does not want to create a recirculator which is unnecessarily long. If one makes a recirculator which works from 4 to $70 \mathrm{GeV}$, for instance, the arcs at $4 \mathrm{GeV}$ will be nearly as long as the arcs at $70 \mathrm{GeV}$, and a substantial number of excess decays will occur. Thus, it is important to create even more recirculator stages. Around a factor of 4 in energy per recirculator seems like a good compromise between decay losses and excess hardware. Thus, a good set of cutoff energies for the recirculators are starting at $4 \mathrm{GeV}$, then $17 \mathrm{GeV}, 70 \mathrm{GeV}, 190 \mathrm{GeV}, 500 \mathrm{GeV}, 2.2 \mathrm{TeV}, 10 \mathrm{TeV}$, $32 \mathrm{TeV}$, and finally up to $100 \mathrm{TeV}$.

The question now becomes how to choose the appropriate RF frequency and number of turns for the recirculators. For low energy recirculators, the length of the recirculator is so short that a kicker to switch from one arc to another would be at best very dificult. Therefore, the low energy recirculators tend to have their number of turns limited by the requirernent that the energy jump should be greater than a few ( 8 is the choice made here) times the RMS energy spread in the beam. This ceases to be an issue in higher energy recirculators.

These issues can be avoided completely if one goes to an Fixed Field Alternating Gradient (FFAG) type of scheme, where a single arc is used for all passes [3]. There are many problems with this type of arc:

- Making the bunch arrive at the right phase of the RF for each pass.

- Achieving a decent dynamic aperture and avoid emittance blowup.

- Creating the complex and often large magnets that are required.

- Creating the required momentum compaction as a function of energy

The first problem, making sure the particles arrive at the correct RF phase, is probably the most difficult of these problems. Possible solutions are to simply supply the required RF power (which may be prohibitively large), to add some ferrite or similar material which can cause the resonant frequency of the cavity to be changed (which may give significant problems with loses and heating, particularly in a superconducting environment), or to use other schemes to vary the resonant frequency of the cavity. 
The next issue becomes the energy spread in the beam, which will turn out to be very large. It turns out that in the low energy recirculators, the energy spread is so large as to require FFAG-like arcs even when individual arcs are used for each pass [3]. These arcs won't have many of the problems of a single-arc design, but are still very complex. It is clearly advantageous to reduce these energy spread if at all possible, if for no other reason than to reduce likely emittance blowup caused by the energy spread. This requirement will tend to push you toward lower frejuency RF and more turns in the recirculator.

However, when one tries to design the arcs, one runs into another problem: the momentum compactions required can easily become too large. Once the momentum compaction becomes above around 0.03 or so, the arcs get very difficult to design [3]. Lowering the moment um compaction tends to push you toward higher freyuency RF and fewer turns in the recirculator.

Higher frequency RF tends to reduce decays (higher gradient), tends to be more efficient (less stored energy), is easier to create power for, and is in general less expensive, but has higher wakefields (which can be a significant problem consid ering the high beam currents under consideration here). Going to more turns will give more decays, requires more arcs in a multiple arc design and therefore is more expensive, but will generally be more efficient both in terms of average power and in terms of linac usage.

Table 3 contains values for recirculator parameters for these schemes. The arcs are assumed to have $2 \mathrm{~T}$ average bending fields. In reality, the arcs may have higher average bending fields in cases where the relative energy spreads are lower it would be nice to take this into account somehow in the computations, but it is unclear how to do so. This is particularly important at higher energies where the arcs get prohibitively long with $2 \mathrm{~T}$ average bend fields (the $2 \mathrm{~T}$ average field is kept nonetheless for comparison purposes only). $L_{\text {orc }}$ is the length of $180^{\circ}$ of arc, and $L_{\text {tinec }}$ is the length of one of the two linac in the recirculator. $P_{\text {peek }}$ is the puwer that must be supplied to replace the energy extracted by the beam at the sume rate the beam is extracting it.

These values were arrived at by various compromises. The values for the lower energy recirculators are often forced. Going to lower frequencies requires momentum compactions that are too high. Going to higher frequencies gives energy spreads which are so large that they don't even allow multiple passes. Generally the number of turns is chosen to be the maximum allowable for passive switching between arcs. Note the large relative energy spreads in these recirculators.

For the higher energy recirculators, there are more choices to be made, and this is reflected in putting multiple lines in the table for a given energy range. The highest frequency given is generally the maximum frequency possible, and the number of turns is the maxinum for that frequency. The relative energy spreads are generally decreasing as we go up in energy, but it might be nice to further decrease the relutive energy sprend so as to make the arcs easier.
TABLE 3. Paramotere for recirculators.

\begin{tabular}{|c|c|c|c|c|c|c|c|c|c|c|c|c|c|}
\hline $\mathbf{V} / \mathbf{c}$ & $\begin{array}{r}p_{\max } \\
\mathrm{GeV} / \mathrm{c} \\
\end{array}$ & M & & $\begin{array}{c}L_{\text {ave }} \\
\text { m }\end{array}$ & in & $\sigma_{r}$ & $\begin{array}{r}\sigma_{\boldsymbol{Z}} \\
\mathrm{MoV} \\
\end{array}$ & $\begin{array}{l}\boldsymbol{P}_{\text {pack }} \\
\text { MW }\end{array}$ & $\begin{array}{c}\text { Decay } \\
\%\end{array}$ & & $\begin{array}{r}\alpha_{C, \text { max }} \\
10^{-3} \\
\end{array}$ & $\begin{array}{r}\sigma_{z} \\
\mathrm{~mm}\end{array}$ & $\begin{array}{c}\pi_{y} \\
m i n\end{array}$ \\
\hline \multicolumn{14}{|c|}{$0.5 \mathrm{TV} / c$ Parameter } \\
\hline 4 & 17 & 400 & 5 & 89 & $\frac{127}{127}$ & 91 & 296 & 1156 & $\overline{3.71}$ & 13 & 14.88 & 9.6 & $\overline{1.5}$ \\
\hline 17 & 70 & 800 & 11 & 367 & 169 & 47 & 579 & 864 & 84 & & 4.04 & 4.3 & 1.0 \\
\hline 70 & 190 & 800 & 12 & 996 & 312 & 37 & 720 & 734 & 1.05 & 54 & 2.63 & 3.4 & 0.9 \\
\hline 70 & 190 & 1600 & 13 & 996 & 231 & 24 & 1143 & 723 & & & 1.05 & 2.2 & 0.7 \\
\hline 190 & 500 & 1600 & 16 & 2620 & 430 & 19 & 1426 & 610 & 4.78 & 84 & 0.68 & 1.7 & 06 \\
\hline 190 & 500 & 3200 & 17 & 2620 & 323 & 12 & 2278 & 595 & 4.85 & 97 & 0.26 & 1.1 & 0.4 \\
\hline \multicolumn{14}{|c|}{$10 \mathrm{TbV} / \mathrm{c}$ Parameters } \\
\hline 4 & 17 & 400 & 6 & 89 & 107 & 92 & 255 & 797 & a. & & 17.7 & $\overline{9.5}$ & $\overline{13}$ \\
\hline 17 & 70 & 800 & 13 & 367 & 144 & 17 & 501 & 575 & 4 & & 4.73 & 4.3 & 10 \\
\hline 70 & 190 & 1600 & 15 & 996 & 200 & 24 & 997 & 482 & 67 & & .21 & 2.1 & 0.6 \\
\hline 190 & 500 & 3200 & 19 & 2620 & 288 & 12 & 2006 . & 404 & $3 s$ & & 0.30 & 1.1 & 0.4 \\
\hline 500 & 2200 & 3200 & 19 & 11527 & 1277 & 7 & 3381 & 503 & 59 & & 0.10 & 0.7 & 0.4 \\
\hline 2200 & 10,000 & 3200 & 19 & 52396 & 5475 & 4 & 5540 & 511 & 5 & & .04 & 0.5 & 0.3 \\
\hline \multicolumn{14}{|c|}{ IeV/c Parameters } \\
\hline 4 & 17 & 200 & 5 & 89 & 179 & 181 & 291 & 186 & 4.6 & & 29.93 & 17.6 & $\overline{0.7}$ \\
\hline 17 & 70 & & & 367 & 238 & 93 & 570 & 153 & & 6 & 8.15 & 8.2 & 0.5 \\
\hline 17 & 70 & 800 & 5 & 367 & 364 & 45 & 1172 & 279 & 0 & 8 & 1.85 & 4.2 & 0.4 \\
\hline 70 & 190 & . & 13 & 6 & 325 & 47 & 1126 & 134 & 4 & 17 & 11 & 4.1 & 03 \\
\hline 70 & & 1600 & & 996 & 490 & 23 & 2305 & 269 & & & 49 & 0 & 0.2 \\
\hline 190 & 500 & 1600 & 17 & & 45 & 23 & 2244 & 114 & 0 & 44 & 0.53 & 1 & 0.2 \\
\hline 190 & 500 & 3200 & & 0 & 67 & 12 & 45 & 226 & 5 & 54 & 0.13 & 1.0 & 0.2 \\
\hline & 0 & & & & 11 & 10 & & 11 & & & & 8 & 0.2 \\
\hline 500 & & & & 11 & a & 6 & 9011 & 114 & 6 & & 0.03 & 5 & 0.1 \\
\hline 2200 & 10,000 & & 23 & 52396 & 3465 & & 14352 & 117 & 6 & & 0.01 & 0.3 & 0.1 \\
\hline, 000 & 32,000 & & & & 9314 & & & 104 & & & 0.01 & 0.1 & 0.1 \\
\hline & & & & & & & & 103 & 6.5 & 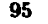 & 0.01 & 0.2 & 0.1 \\
\hline
\end{tabular}

Thus, for the $0.5 \mathrm{TeV} / \mathrm{c}$ parameters, a lower frequency solution is given when possible, with the nuriber of turns chosen to have about as many decays as in the higher frequency case. Note that the linac tends to get longer; this is probsably the primary cost of going to the lower frequency. There is also a slight decrease in efficiency, which is significant but not terribly so. You are trading off the cost of the linac (including its associated power) with the complexity of the arcs by changing the frequency of the linacs.

The first four energy ranges for the $0.5 \mathrm{TeV}$ and the $10 \mathrm{TeV}$ parameters are similar except for a small (leas than 15\%) change in the longitudinal emittance (the transverse emittance also decreases somewhat). Note that substantial change that can potentially occur in the linac length, energy spread, and peak powe requirement as a result. Thus, particularly for the lower energy recirculators, a rexluced longitudinal enittance can be of significant advantage in cost savings.

For the $10 \mathrm{TeV}$ parameters, it turns out that an RF frequency of $6.4 \mathrm{GHz}$ is not workable since beam loading becomes too high with $3 \times 10^{12}$ particles per bunch (Eq. (34) gives a value greater than 1). Thus, for higher energies we continue to 
us: $3.2 \mathrm{GHz} \overline{\mathrm{R}} \mathrm{F}$. In reality, one can use an aimost arbitrary number of turns at the ligher energies (above $500 \mathrm{GeV}$ ), since the relative energy spreads are relatively low (allowing passive switching), and in any case active switching is probably possible since the rings are much longer. 19 turns was chosen arbitrarily, basically to be expral to the number of turns in the $190 \mathrm{GeV} / \mathrm{c}$ to $500 \mathrm{GeV} / \mathrm{c}$ recirculator. More turns requires more hardware in a multiple arc system. The decay losses start to rise in the higher energy machines due to the increasing length of the linac. The relative small $\alpha_{C}$ suggests that it may be advantageous to increase the number of sides in the recircuiator, allowing a greater synchrotron tune in the recircuiator ( 0.15 per side) and a correspondingly smaller energy spread. The bunch lengths that come out of the caicuiation are also extremeiy shori, and that is another indicator that it would be helpful to increase the synchrotron tune in this fashion. Many-sided designs wiil be considered in future work.

For the $100 \mathrm{TeV} / c$ parameters, the longitudinal emittance has increased substantially, and so lower frequencies are often required than are required in the other cases. However, for some of the lower energies, we have provided a higher frequency solution that has a larger energy spread. The relative energy spread is still smaller than it was for the previous stage, so the arcs would be no worse than the arcs in the earlier stages. However, it would be nice to take advantage of the lower energy spreads to construct simpler arcs.

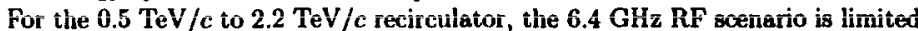
to 23 turns for passive switching, but the maximum number of turns for the $3.2 \mathrm{GHz}$ $\mathrm{RF}$ scenario is much higher. Assuming we want to limit the number of turns to limit decays and arc complexity, I have chosen to use a maximum of 23 turns here and for subsequent recirculators. Also, it turns out that going above $6.4 \mathrm{GHz}$ RF leads to beam loading problems like those for the $10 \mathrm{TeV} / c$ case, and thus we will limit ourselves to this frequency.

\section{Arc Design}

Clearly one of the greatest challenges lies in the design of the arcs for these recirculating accelerators. The arcs for the low energy systems must accept rather large relative energy spreads, and this is the primary challenge. Arc designs for these large energy acceptances are being considered by several people, including Al Garren, Carol Jolustone, Dejan Trbojevic, Weishi Wan. In addition, these same people are studying single-arc designs where the entire energy range of the recirculator passes through a single arc. It may even end up making sense to have a sinall uumber of arcs, where the beam passes through each arc a few times. One of the greatest difficulties in these designs is meeting the requirements on momentum compaction that come from longitudinal cousiderations, and many (but not all) of these designs have yet to address this issue.

It would be particularly useful to get some kind of rough parameterization of how the dipole packing fraction and other parameters behave with respect to design constraints put on the arcs.

Don Summers has come up with an idea for a different geometry for the recirculating accelerator, which has been called a "dogbone geometry." The idea is to have a single linac through which the particles pass, and have arcs on the end of that linac which return the particles into the same linac. The advantage of this scheme is that lower energy particles can go through a shorter arc than the high energy particles, since the length of the arc is not determined by the distance between linacs. Such a scheme can aliow one to rediuce decays and/or reduce linac costs in the recirculator. There are issues related to supplying RF power due to the asymmetric way in which the bunches would pass througin such a system, and such a system cannot be expanded to many sides for the high energy recirculators, but is certainly an attractive possibility for the low energy recircuiators.

At higher energies scheme with ramping magnets has been considered for the arcs [2]. While superconducting magnets cannot be ramped fast enough, it is possible that normal conducting magnets could be. So a hybrid scheme is user consisting of interieaved fixed-field superconducting magnets and puised normalconducting magnets. Such a scheme has yet to be examined carefully, in particular the nature of the orbits has yet to be convidered. It will have similar difficulties to FFAG systems due to large orbit swings, but in principle it should be better since there is an extra degree of contiol in the ability to ramp some of the magnets.

Arc designs will not be discussed in much more detail here. Their design is progressing, and the status and other issues with their design will be reported on in the future.

\section{Other Issues}

It is possible to use isochronous designs for the recirculators instead of these designs with a finite synchrotron tune. The isochronous designs lack many of the advantages of the non-isochronous designs: energy spreads will be larger, collective instabilities are more difficult to control, and there may be difficulties preserving polarization. In addition, an isochronous design will necessarily increase the longitudinal beam emittance, which is already problematically large (this effect is particularly significant at the lower energiea). Isochronous designs do have the advantage that they eliminate one of the primary difficulties associated with single-arc recirculator, the issue of making the beam arrive at the correct phase of the RF. Isochronous design principles will not be discussed here, but will be treated in a subsequent paper.

Wakefields and their effects have yet to be computed for these kinds of systems, but it is clear that they will be a significant effect, due to the large beam current. At the higher energies, it is important to come up with a scheme which has longer bunch lengths, since the extremely short bunch lengths given in Tab. 3 will create 
substantial wakefield effects. Going to many-sided designs should be considered for these high energies, not only because of the short bunch lengths but also because the synchrotron tune will be larger and therefore will more readily be able to correct the strong wakefield effects that will occur.

\section{CONCLUSION}

We have described a method for designing the acceleration systems for a muon collider. The design method is based primarily on considering the longitudinal phase space dynamics of the bunch. The muons are initially accelerated in a linac which most likely contain several frequencies of RF, and then are accelerated by a series of recirculating accelerators. We have laid out what the parameters might look like for various future high-energy muon colliders, and discussed some of the tradeoffs involved.

The relatively large longitudinal emittances in a muon collider create significan difficulties at lower energies. It requires low-frequency RF in the linac that initially accelerates the muons after the cooling stage, and also requires large energy acceptance arcs in the early stages of recirculation.

Much work remains to be done. The studies of the arcs in the recirculators need to be continued and expanded. The effect of wakefields needs to be considered. Linac designs need to be looked at to determine, achievable parameters as well as wakefields. Multi-sided recirculator designs should be looked at, particularly for high energies. Finally, much more work needs to be put into optimization of these lesigns for cost and performanco.

We have laid out a set of parameters for a high-energy muon collider which does not appear to be unrealistic. With these parameters as a starting point, it is possible to attempt to estimate the cost of the acceleration for a high-energy muon collider, and from that determine what dircction one should go to reduce those costs.

\section{ACKNOWLEDGEMENTS}

This research has been supported in part by the U.S. Department of Energy under contract no. DE-AC02-98CH10886.

\section{REFERENCES}

(I) Brure King, parameters supplied for this conference.

[2] C'murles M1. Aukenbrandt et al. (Muon Collider Collaboration), Phys. Rev. ST Accel. Beams 2 , (281001 (1999)
(3) Carol Johnstone, privete communication.

NS-30, 2669-2771 (1983)

Alain Blondel, talk given at the Noutrino Factory and Muon Collider Collaboration Meeting, A 